\title{
Análisis de armónicos de un motor trifásico mediante transformadas integrales para analizar fallas de funcionamiento
}

\author{
Harmonic analysis of a three-phase motor through integral transforms to \\ analyze malfunctions
}

Wilson Marcelo Román Vargas. ${ }^{1}$, Norma del Pilar Barreno Layedra. ${ }^{2}$, Juan Manuel Martínez Nogales. ${ }^{3} \&$ Jorge Cachuput Gusñay. ${ }^{4}$

Recibido: 14-07-2019 / Revisado: 23-07-2019 /Aceptado: 15-08-2019/ Publicado: 10-09-2019

\begin{abstract}
.
DOI: https://doi.org/10.33262/cienciadigital.v3i3.4..869

The research establishes a mathematical modeling of the study of the harmonics produced in the stator currents of a three-phase squirrel cage motor, considering that the information contained in the signal harmonics can indicate the presence of various types of both electrical and mechanical failures, To perform the simulation of the mathematical model, Matlab Simulink software was used, the assessment of the mathematical model is given experimentally by correlating the total harmonic distortion value of the current signal of each phase of the stator obtained in the simulation and comparing with the values established by the CONELEC entity in charge of determining the limit values that a current signal must have in order for a motor to be functioning correctly and not to have any electrical and / or mechanical failure; the analysis of variance was implemented accompanied by the Tuckey test for the evaluation of results obtaining a difference of $2 \%$ between the determined model and an engine that is in optimal operating conditions.

Keywords: Harmonic analysis, squirrel cage motor, Simulink, Fourier Transform Resumen.

La investigación establece una modelación matemática del estudio de los armónicos producidos en las corrientes del estator de un motor trifásico jaula de ardilla,

${ }^{1}$ Universidad de Fuerzas Armadas ESPE-Sangolquí, Departamento de Ciencias Exactas, Sangolquí, Ecuador, wmroman@espe.edu.ec

${ }^{2}$ Universidad de Fuerzas Armadas ESPE-Sangolquí, Departamento de Ciencias Exactas Sangolquí, Ecuador.npbarreno@espe.edu.ec

3 Escuela Superior Politécnica de Chimborazo, Facultad de Recursos Naturales. Riobamba, Ecuador, jumartinez@espoch.edu.ec

4 Escuela Superior Politécnica de Chimborazo, Facultad de Recursos Naturales. Riobamba, Ecuador, jorge.cachuput@espoch.edu.ec
\end{abstract}


considerando que la información contenida en los armónicos de la señal pueden indicar la presencia de diversos tipos de fallas tanto eléctricas como mecánicas, para realizar la simulación del modelo matemático se utilizó el software de Simulink de Matlab, la valoración del modelo matemático esta dado de forma experimental al correlacionar el valor de distorsión armónica total de la señal de corriente de cada fase del estator obtenida en la simulación y comparar con los valores establecidos por el CONELEC entidad encargada de determinar los valores límites que debe tener una señal de corriente para que un motor esté funcionando correctamente y no tenga ninguna falla eléctrica y/o mecánica; se implementó el análisis de varianza acompañado de la prueba de Tuckey para la valoración de resultados obteniendo una diferencia del $2 \%$ entre el modelo determinado y un motor que se encuentra en condiciones óptimas de funcionamiento.

Palabras claves: Análisis armónico, motor jaula de ardilla, Simulink, Transformada de Fourier.

\section{Introducción}

Los motores eléctricos jaula de ardilla tienen una gran presencia en la automatización de los procesos industriales, donde su consumo eléctrico esta dado aproximadamente en un $63 \%$ de la energía eléctrica producida según [1], el estudio realizado en [2] determina que una de las técnicas de mantenimiento predictivo para determinar el estado técnico de las máquinas rotatorias esta dado en función del análisis de los armónicos producidos por los motores, su mal funcionamiento implica un incremento en el consumo de energía, el estado de carga y la temperatura de los rodamientos del motor, el trabajo realizado por [3] determina que los componentes armónicos constituyen parámetros importantes para el diagnóstico de falla del sistema de rotor, en este contexto, la presente investigación desarrolla la modelación y simulación de un motor trifásico jaula de ardilla en Simulink para detectar fallas mediante el análisis de armónicos utilizando la Transformada de Fourier, la misma que nos permite extraer de forma eficiente y eficaz los componentes armónicos del sistema del rotor.

El estudio realizado por [4] muestra el análisis comparativo entre un motor de inducción jaula de ardilla trifásico tradicional y un motor de inducción trifásico asimétrico alimentado por voltaje monofásico, referenciando este trabajo se determina a la variación del voltaje, frecuencia y velocidad del motor como los parámetros que son analizados en la presente investigación, en [5] se muestra el estudio para la evaluación y clasificación de fallas en el aislamiento eléctrico de los motores de inducción y [6] investiga los diferentes factores de desequilibrio utilizados para los motores de inducción, estas investigaciones promueven utilizar las diferentes herramientas tecnológicas para realizar investigaciones sobre problemas de motores.

En tal virtud, la presente investigación es de carácter experimental comparativo, donde se varían los diferentes parámetros citados anteriormente con la intención de analizar el comportamiento del motor y verificar que los resultados obtenidos se enmarquen en las especificaciones técnicas de funcionamiento dados por los fabricantes y controlados por el 
CONELEC, la modelación se realiza considerando el análisis de Fourier, para lo cual, se obtiene la expansión en Serie de Fourier en su forma trigonométrica y compleja de la señal continua $\mathrm{x}(\mathrm{t})$, se aplica el cálculo numérico ante la dificulta de resolver de forma analítica los diferentes integrales de la onda, por lo que es necesario realizar la discretización de la señal mediante la Trasformada de Fourier lo cual se logra realizando un muestreo del plano tiempofrecuencia obteniendo la Transformada Discreta de Fourier la misma que esta implementada en Matlab mediante la función fft, y su algoritmo reduce el tiempo de cálculo de $n^{2}$ pasos a $n \log _{2}(n)$, la eficiencia del algoritmo requiere considerar una muestra de $2^{n}$ puntos de la serie.

Los armónicos son ondas que se presentan tanto en tensión como en corriente y afecta a la calidad de la potencia eléctrica[7], e influye directamente en el rendimiento y vida útil del equipo eléctrico [8], específicamente al motor al cual se le proporciona energía eléctrica, se analiza el fenómeno de la distorsión armónica en un motor de inducción trifásico jaula de ardilla, donde los motores de inducción constituyen dispositivos de accionamiento muy empleados en el mundo industrial [9], el análisis de este tipo de fenómenos resulta un poco complicado de realizarse manualmente pero existen herramientas informáticas e incluso software y hardware especializado para su análisis y monitoreo constante, por esto [10] nos muestras las ventajas de la utilización de software muy fuerte en este tipo de análisis como Matlab, específicamente con la aplicación Simulink que es considerado como la herramienta más fuerte y con mayores aplicaciones en simulaciones y cálculo de todo índole.

Este articulo presenta una metodología que emplea de forma directa el uso de software matemático para una mayor facilidad de cálculo y de gráficas en comparación de los métodos convencionales, ahorrando mucho tiempo al análisis de los armónicos presentes en motores de inducción trifásicos. La metodología se fundamenta en el análisis experimental de las corrientes armónicas producidas en un motor de inducción trifásico desde el momento en que se estabiliza y el mismo funciona a velocidad, intensidad y corriente nominal para evitar así datos del arranque del motor en donde los datos son muy diferentes a cuando el motor funciona en estado nominal, del desarrollo experimental se determina la relación entre el orden de armónicos utilizados y el margen de error originado.

\section{Metodología}

\section{A. Armónicos}

Los armónicos se definen habitualmente con los dos datos más importantes que les caracterizan, que son: La amplitud la misma que hace referencia al valor de la tensión o intensidad del armónico y el orden que hace referencia al valor de su frecuencia referido a la fundamental $(50 \mathrm{~Hz})$. Así, un armónico de orden 3 tiene una frecuencia 3 veces superior a la fundamental, es decir $3 * 50 \mathrm{~Hz}=150 \mathrm{~Hz}$ [11]. En la Figura 1, se visualiza los parámetros ingresados en el Simulink para representar la forma de la onda sin contenido de armónicos, con una frecuencia constante de $60 \mathrm{~Hz}$ y una amplitud constante de 1 pu. 


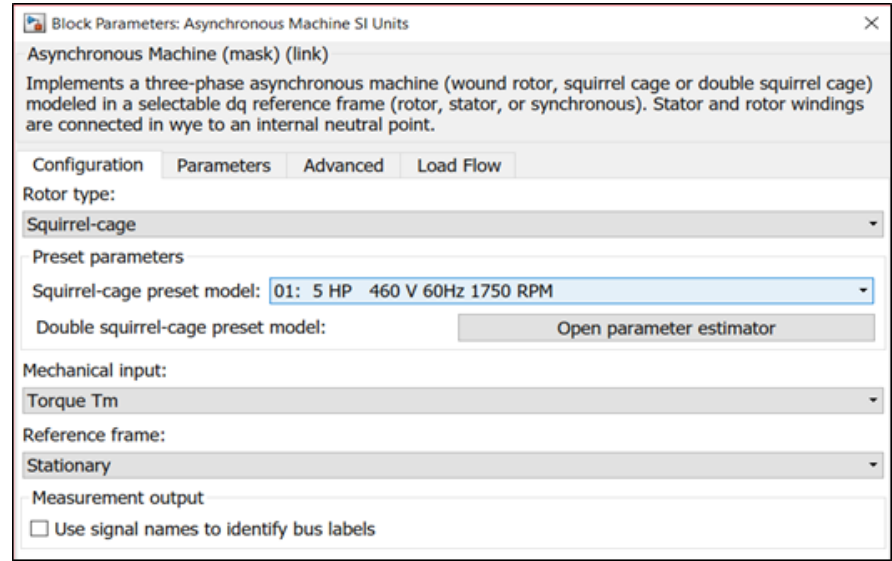

Figura 1 Valores del motor de inducción jaula de ardilla en Simulink

La frecuencia de la onda periódica se denomina frecuencia fundamental y los armónicos son señales cuya frecuencia es un múltiplo entero de esta frecuencia. Cuando una onda periódica no tiene esta forma sinusoidal se dice que tiene contenido armónico, lo cual puede alterar su valor pico y/o valor RMS causando alteraciones en el funcionamiento normal de los equipos que estén sometidos a esta tensión o corriente. La distorsión armónica total (THD) [11] va ser fundamental para el análisis de fallas mecánicas o eléctricas en el motor jaula de ardilla ya que es una herramienta para definir los efectos de los armónicos en el motor y así poder comparar en base a los valores establecidos por la CONELEC para que un motor este en correcto funcionamiento.

Frecuencia fundamental (f 1): Frecuencia de la onda original $(50 / 60 \mathrm{~Hz})$

- Orden de un armónico (n): Número entero dado por la relación de la frecuencia de un armónico a la frecuencia fundamental. Con el orden se determina la frecuencia del armónico (Ejemplo: $5^{\circ}$ armónico $\rightarrow 5 \bullet 50 \mathrm{~Hz}=250 \mathrm{~Hz}$ ). [10]

- Tasa de distorsión armónica (THD) [11]: Relación entre el valor eficaz del residuo armónico de la tensión y/o corriente y el valor de la componente fundamental.

La medición y la resultante de los armónicos se explica por la distorsión armónica total (THD: Total Harmonics Distortion), el método de cálculo se fundamenta en la utilización de la norma CEI 61000-2-2 [11] donde define el THDF como la relación (en porcentajes) entre el valor eficaz de las componentes armónicas y la amplitud de la armónica fundamental

$$
T H D_{F}=\frac{\sqrt{U_{2}^{2}+U_{3}^{2}+U_{5}^{2}+. .}}{U_{1}^{2}}
$$

Donde:

U representa los armónicos

B. El análisis de Fourier en el estudio de armónicos producidos por un motor

En [12], menciona que la transformada de Fourier rápida se usa para cambiar entre dominios espaciales y de frecuencia, e indica que este procedimiento se puede aplicar para simular máquinas de inducción con fallas de bobinado, en [13] se determina la relación de conmutación a frecuencias fundamentales entre el contenido armónico más alto y más bajo 
como un criterio de distorsión armónica, en el trabajo publicado en [14] se encuentra la formulación del Teorema de Fourier, la ecuación (2) indica que toda onda periódica no sinusoidal puede ser descompuesta como la suma de ondas sinusoidales, mediante la aplicación de la serie de Fourier, siempre y cuando se cumplan las siguientes condiciones:

- Que la integral a lo largo de un periodo de la función sea un valor finito,

- Que la función posea un número finito de discontinuidades en un periodo, y

- Que la función posea un número finito de máximos y mínimos en un periodo.

Cualquier función $f(t)$ con periodo $2 \pi$ tiene su representación en series de Fourier de acuerdo con la siguiente expresión:

$$
f(t)=\frac{1}{2} a_{0}+\sum_{n=1}^{+\infty} a_{n} \cos (n \omega t)+\sum_{n=1}^{+\infty} b_{n} \operatorname{sen}(n \omega t)
$$

Donde $a_{0}, a_{n} y b_{n}$ representan los coeficientes de la expansión en Serie de Fourier para la función $f(t)$ dado en su forma trigonométrica.

Para el análisis espectral de las ondas es necesario definir la Transformada de Fourier de la señal continua $x(t)$ dada por

$$
F\{x(t)\}=X(i \omega)=\int_{-\infty}^{+\infty} x(t) e^{-j \omega t} d t
$$

Donde la ecuación (3) representa la suma de funciones exponenciales complejas periódicas [15]; la Transformada Discreta de Fourier se define como un operador lineal que actúa sobre un vector de entrada $x_{N}$, de $N$ muestras en el dominio de tiempo discreto y que genera coeficientes $X_{N}$ de longitud $N$, la misma que está dado por

$$
X_{N}=F_{N} x_{N}
$$

La transformada rápida de Fourier está dada por

$$
X(k)=\sum_{j=1}^{N} x(j) \omega_{N}^{(j-1)(k-1)}
$$

La transformada inversa rápida de Fourier está dada por

$$
\begin{array}{r}
x(j)=\frac{1}{N} \sum_{k=1}^{N} x(k) \omega_{N}^{-(j-1)(k-1)} \\
\operatorname{Con} \omega_{N}=\exp \left(-\frac{2 \pi i}{N}\right)
\end{array}
$$

Para [16], la necesidad de utilizar los algoritmos computacionales de las ecuaciones 4, 5, y 6; están dadas en la necesidad de desarrollar técnicas avanzadas de diagnóstico de máquinas eléctricas.

La Transformada de Fourier en tiempo corto, el método consiste en dividir la señal no estacionaria en pequeños intervalos donde se supone que la señal es estacionaria, mediante una función ventana de anchura determinada, que se desplaza y se multiplica con la señal, al aplicarse la DFT en cada intervalo se obtiene una distribución tiempo-frecuencia de la señal, 
este proceso se formula mediante

$$
\begin{aligned}
\operatorname{STFT}_{x}^{\text {window }}( & (t i, \omega) \\
& =\int_{t}[x(t) \cdot \text { window }(t-t i)] \cdot e^{-j \omega t} d t
\end{aligned}
$$

De esta forma se pretende abordar el problema de incertidumbre de Heisenberg [17], en cual manifiesta que no es posible la representación exacta de una señal dada en tiempo-frecuencia, salvo se considere intervalos de tiempo en las cuales existan determinadas bandas de frecuencia.

C. Simulación del motor de inducción

El software de simulación representa una herramienta importante para desarrollar controladores, simplificando la obtención de los parámetros de este y además permitiendo visualizar el comportamiento del sistema para diferentes valores del controlador. Se utiliza como herramienta para la simulación, análisis y obtención de parámetros el Simulink de Matlab [18], debido a que cuenta con una amplia gama de herramientas para el diseño de sistemas dinámicos, ya sea en función del tiempo o en función de la frecuencia (transformada de Fourier rápida).

En la Figura 2, se muestra el diseño del motor de inducción seleccionado para trabajar, el cual tiene un voltaje nominal de $460 \mathrm{~V}$, una frecuencia de $60 \mathrm{~Hz}$ que es la frecuencia con la que se maneja la transmisión ecuatoriana de energía eléctrica y la velocidad nominal que trabaja el motor es de 1750 RPM, además se presenta el modo de conexión de un motor de inducción en Simulink para analizar sus armónicos en estator y en el rotor.

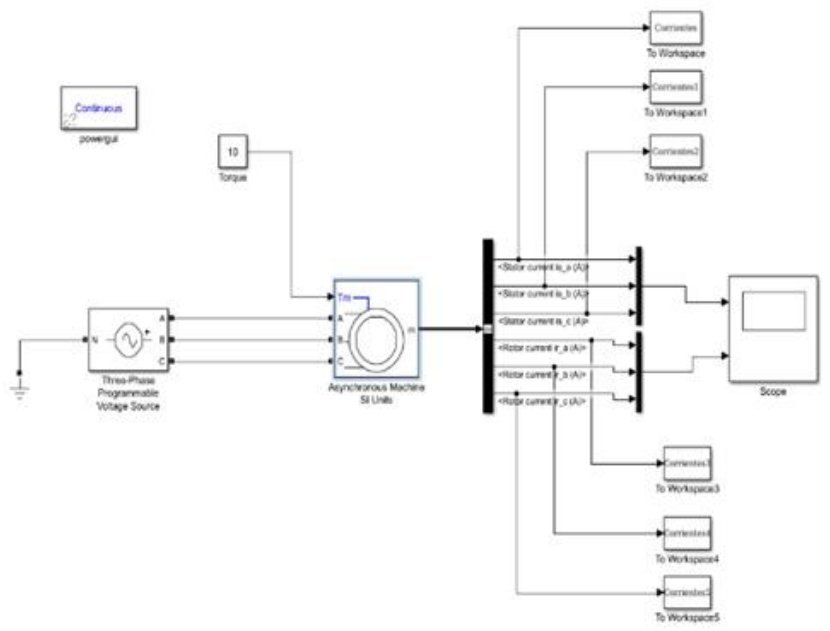

\section{Resultados}

Figura 2 Motor de inducción jaula de ardilla en Simulink

Los datos de corriente en el estator por fase, el THD por fase y los parámetros mecánicos [20] son extrapolados y vectorizados a través del Matlab para aplicar los análisis estadísticos descriptivos que permitan definir intervalos de confianza para los errores detectados a la 
variación de diferentes armónicos.

A. Señales de las corrientes de estator

La señal de la figura 3 muestra la corriente de las fases del estator con respecto al tiempo como podemos observar al inicio de la señal hay una distorsión lo cual se produce por el arranque del motor y luego se estabiliza llegando al valor nominal de corriente consumido por el motor [21]. Se procede analizar las señales del estator por fase mediante la FFT análisis (transformada rápida de Fourier), con armónicos y sin armónicos.

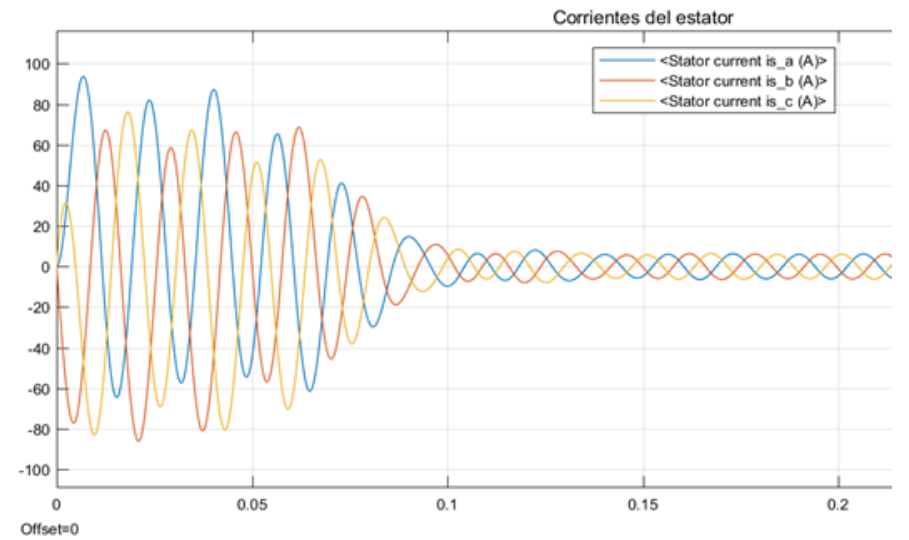

Figura 3 Señales de corriente de las fases del estator

B. Señal de corriente sin armónicos

En la figura 4 observamos una señal de corriente sinusoidal pura que es producida por la fuente de alimentación trifásica de la fase B, la cual no contiene armónicos y esta señal la analizamos en 11 ciclos con una frecuencia fundamental de $60 \mathrm{HZ}$ para hallar su FFT y observar el comportamiento de los armónicos en la señal. En este análisis también nos brinda información del THD que es igual a $0.56 \%$.

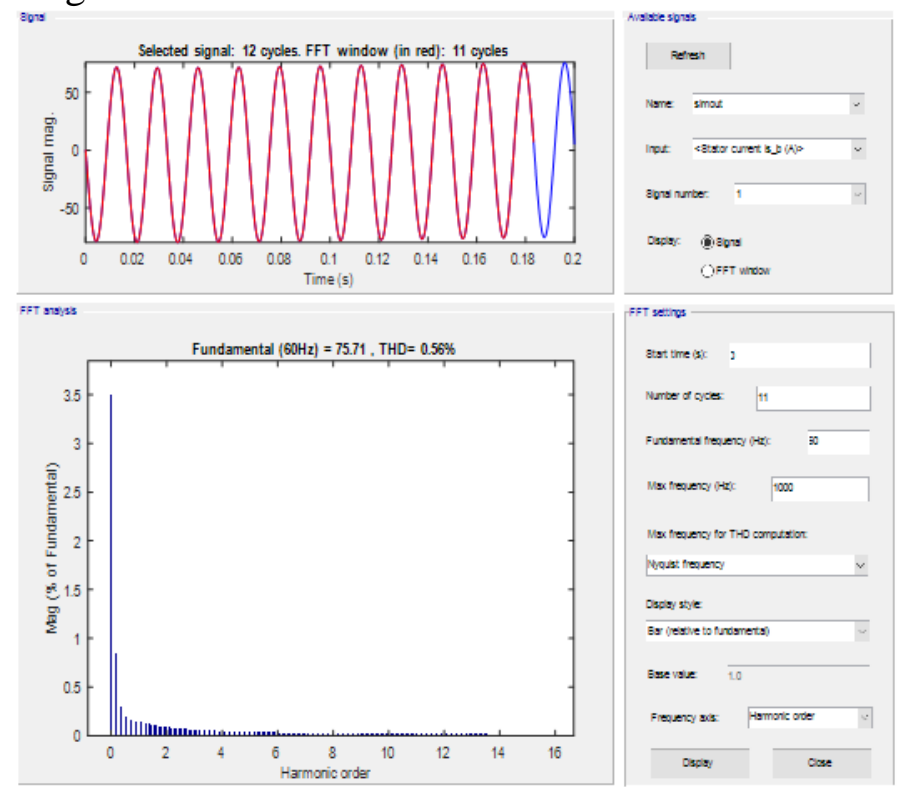

Figura 4 Señal de corriente pura del estator de la fase B con voltaje nominal y su FFT 
C. Señal con armónicos con voltaje nominal (460v)

Corriente de la fase A del estator

En la figura 5 observamos una señal de corriente producida por la fase A del estator con distorsión al inicio esto es debido al arranque del motor y luego se estabiliza debido al comportamiento normal del mismo, la señal se analiza desde que se estabiliza a los 0.13 (s) en 7 ciclos con una frecuencia fundamental de $60 \mathrm{~Hz}$ para hallar su FFT y observar el comportamiento de los armónicos en la señal. En este análisis también nos brinda información del THD que es igual a 4.53\%, lo cual nos indica un aumento de armónicos que sería causante de pérdidas en el motor.

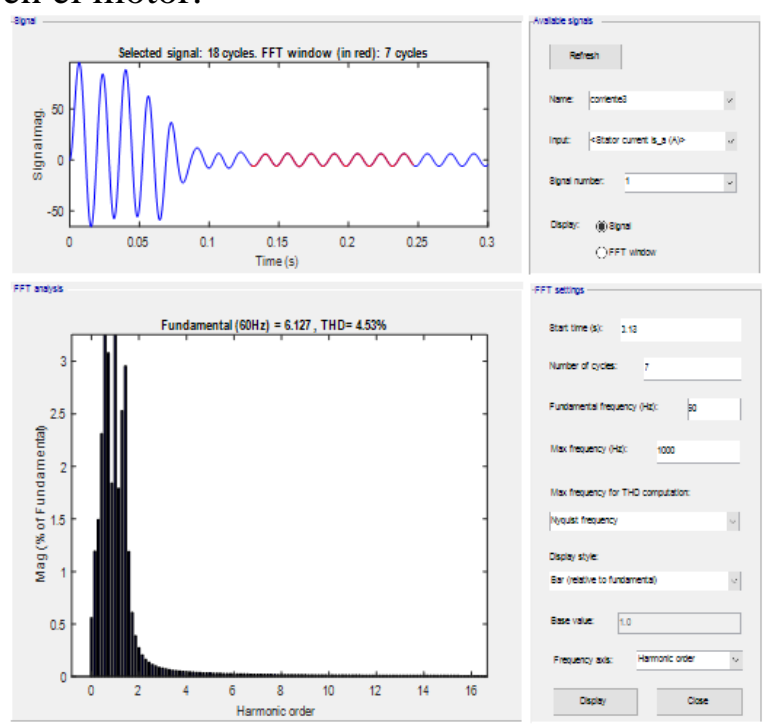

Figura 5 Señal de corriente del estator de la fase A con voltaje nominal y su FFT Corriente de la fase B del estator

En la figura 6 observamos una señal de corriente producida por la fase B del estator con distorsión al inicio esto es debido al arranque del motor y luego se estabiliza debido al comportamiento normal del mismo, la señal se analiza desde que se estabiliza a los 0.13 (s) en 7 ciclos con una frecuencia fundamental de $60 \mathrm{~Hz}$ para hallar su FFT y observar el comportamiento de los armónicos en la señal. En este análisis también nos brinda información del THD que es igual a 4.74\%, lo cual nos indica un aumento de armónicos que sería causante de pérdidas en el motor.

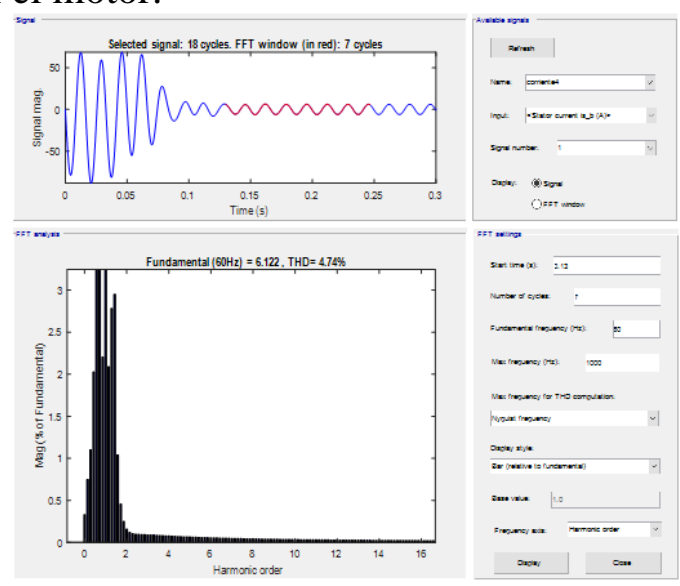

Figura 6 Señal de corriente del estator de la fase B con voltaje nominal y su FFT 


\section{Corriente de la fase $\mathrm{C}$ del estator}

En la figura 7 observamos una señal de corriente producida por la fase $\mathrm{C}$ del estator con distorsión al inicio esto es debido al arranque del motor y luego se estabiliza debido al comportamiento normal del mismo, la señal se analiza desde que se estabiliza a los 0.13 (s) en 7 ciclos con una frecuencia fundamental de $60 \mathrm{~Hz}$ para hallar su FFT y observar el comportamiento de los armónicos en la señal. En este análisis también nos brinda información del THD que es igual a 4.99\%, lo cual nos indica un aumento de armónicos que sería causante de pérdidas en el motor.

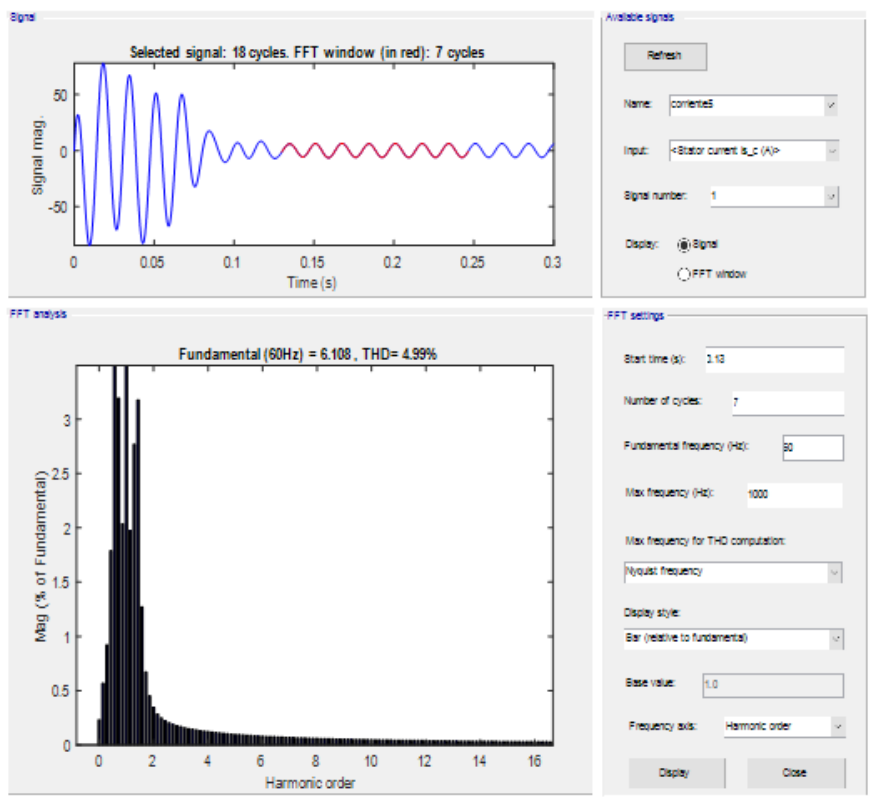

Figura 7. Señal de corriente del estator de la fase $\mathrm{C}$ con voltaje nominal y su FFT

Los resultados de la experimentación se muestran en la tabla 1, los armónicos se clasifican según su amplitud (indicada en \% con respecto a la fundamental) y señales con armónicos a $450 \mathrm{~V}$ y $460 \mathrm{~V}$.

Tabla 1. Diferencia de señal pura y con armónicos

\begin{tabular}{cccc}
\hline FASE & Señal pura & $\begin{array}{c}\text { Señal con } \\
\text { armónicos a } \\
\mathbf{4 6 0 V}\end{array}$ & $\begin{array}{c}\text { Señal con } \\
\text { armónicos a } \\
\mathbf{4 5 0 V}\end{array}$ \\
\hline FASE A & $0.56 \%$ & $4.53 \%$ & $4.36 \%$ \\
FASE B & $0.56 \%$ & $4.74 \%$ & $4.26 \%$ \\
FASE C & $0.56 \%$ & $4.99 \%$ & $4.26 \%$ \\
\hline
\end{tabular}

Tomando en cuenta los valores permitidos por el CONELEC sobre la calidad de distribución de servicio eléctrico del Ecuador, podemos observar que los valores máximos de la tabla 2 son inferiores a los valores obtenidos de la simulación, tomando en cuenta que por la situación de la estabilidad de las ondas después del arranque del motor solo se analiza 7 armónicos a partir de que se encuentre en estado estable el motor. 
Tabla 2. Límites de distorsión armónica máxima permisible en corriente para sistemas de $120 \mathrm{~V}$ a $69,000 \mathrm{~V}$ [22]

\begin{tabular}{|c|c|c|c|c|c|c|}
\hline \multirow[b]{2}{*}{ RELACIÓO $_{I_{s c} / I_{L}}$} & \multicolumn{5}{|c|}{$\begin{array}{c}\text { LIMITES PARA } \\
\text { COMPONENETES ARMÓNICAS } \\
\text { IMPARES EN \% DE }\end{array}$} & \multirow{2}{*}{$\begin{array}{c}\text { DISTORSIÓN DE } \\
\text { DEMANDA TOTAL EN \% } \\
(\% \text { TDD })\end{array}$} \\
\hline & $\begin{array}{c}3- \\
11\end{array}$ & $\begin{array}{c}11- \\
17\end{array}$ & $\begin{array}{l}17- \\
23 \\
\end{array}$ & $\begin{array}{l}23- \\
35 \\
\end{array}$ & $35-51$ & \\
\hline$I_{S C} / I_{L}<20$ & 4.0 & 2.0 & 1.5 & 0.6 & 0.3 & 5.0 \\
\hline$I_{S C} / I_{L}$ & 7.0 & 3.5 & 2.5 & 1.0 & 0.5 & 8.0 \\
\hline$<50$ & & & & & & \\
\hline $\begin{aligned} I_{S C} / I_{L} & \\
& 50< \\
& <100\end{aligned}$ & 10.0 & 4.0 & 4.0 & 1.5 & 0.7 & 12.0 \\
\hline $\begin{array}{r}I_{S C} / I_{L} \\
100< \\
<1000\end{array}$ & 12.0 & 5.5 & 5.0 & 2.0 & 1.0 & 15.0 \\
\hline$\stackrel{I_{S C} / I_{L}}{ }>1000$ & 15.0 & 7.0 & 6.0 & 2.5 & 1.4 & 20.0 \\
\hline
\end{tabular}

Para la Fase A, el máximo sugerido por el CONELEC es de $4 \%$ mientras que los datos arrojados nos dan un $4.53 \%$ a una tensión de $460 \mathrm{~V}$.

Para la Fase A, el máximo sugerido por el CONELEC es de $4 \%$ mientras que los datos arrojados nos dan un $4.36 \%$ a una tensión de $450 \mathrm{v}$, tomando $450 \mathrm{~V}$ como la variación mínima de tensión en la red eléctrica.

Para la Fase B, el máximo sugerido por el CONELEC es de $4 \%$ mientras que los datos arrojados nos dan un $4.74 \%$ a una tensión de $460 \mathrm{~V}$.

Para la Fase B, el máximo sugerido por el CONELEC es de $4 \%$ mientras que los datos arrojados nos dan un $4.26 \%$ a una tensión de $450 \mathrm{~V}$, tomando $450 \mathrm{~V}$ como la variación mínima de tensión en la red eléctrica.

Para la Fase C, el máximo sugerido por el CONELEC es de $4 \%$ mientras que los datos arrojados nos dan un $4.99 \%$ a una tensión de $460 \mathrm{~V}$.

Para la Fase C, el máximo sugerido por el CONELEC es de $4 \%$ mientras que los datos arrojados nos dan un $4.26 \%$ a una tensión de $450 \mathrm{~V}$, tomando $450 \mathrm{~V}$ como la variación mínima de tensión en la red eléctrica.

En la tabla 3 se presenta el resumen del análisis realizado determinando intervalos de confianza para los índices de tensión y su correspondiente perturbación producida. 
Tabla 3 Intervalos de confianza para los índices de tensión

\begin{tabular}{ll}
\multicolumn{1}{c}{ ÍNDICE DE } & \multicolumn{1}{c}{$\begin{array}{c}\text { PERTURBACIÓN } \\
\text { TENSIÓN }\end{array}$} \\
\hline $\mathrm{THD}_{\mathrm{F}}<5 \%$ & No existe perturbación \\
$5 \%<\mathrm{THD}_{\mathrm{F}}<10 \%$ & Probablemente mal funcionamiento \\
$\mathrm{THD}_{\mathrm{F}}>10 \%$ & Seguro mal funcionamiento \\
\hline
\end{tabular}

Para la verificación de la hipótesis entre la comprobación de las medias de los armónicos producidos por la simulación del modelo matemático y los valores medios medidos en el motor se obtiene un p-valor de 0.6862 que es superior al valor de 0.05 ; por lo tanto, no se rechaza $H_{0}$ y no se obtiene resultados de un post-hoc que surgen cuando se rechaza $H_{0}$, esto quiere decir que los armónicos producidos por el modelo matemático son virtualmente iguales a los valores reales.

\section{Conclusiones}

Utilizando los algoritmos del análisis de Fourier implementados en el software Matlab y con la utilización del Simulink se validó de forma experimental la variación en las señales de corriente de las fases para un motor trifásico jaula de ardilla con el fin de conocer la distorsión armónica total (THD), en la simulación se ha logrado determinar los intervalos de variación se encuentra entre el $1 \%$ y $4 \%$ considerado como un funcionamiento normal del motor, pero un porcentaje superior al $5 \%$ representa un indicador de la presencia de fallas eléctricas o mecánicas en el motor. Además, por el método de superposición se determinó que la presencia de los armónicos de mayor orden determina el comportamiento general de un motor, de esta forma se puede simular el efecto de cada armónico presente en la onda que se analiza sobre el comportamiento del motor, que, desde el punto de vista didáctico, permite comparar las características de la máquina sobre la influencia de los diferentes armónicos de tiempo.

La determinación del modelo matemático y su implementación en el Simulink de Matlab permite contar con una herramienta didáctica que facilite el estudio de los armónicos de un motor, en el mismo que se puede ejecutar diferentes variaciones a los parámetros estudiados, sin que éste hecho afecte significativamente las condiciones físicas de un motor de laboratorio.

\section{Referencias bibliográficas.}

[1] Conelec, P. M. D. E. (2013). Electrificación 2013-2022. Anexo A: Metodología de la Proyección de la Demanda.

[2] Torres-Rodríguez, R. M., \& Batista-Rodríguez, C. R. (2010). Análisis vibro dinámico de motores eléctricos. Ingeniería Mecánica, 13(1), 9-18.

[3] Yao, J., Tang, B., \& Zhao, J. (2016). Improved discrete Fourier transform algorithm for harmonic analysis of rotor system. Measurement, 83, 57-71.

[4] Wellington, P., de Mendonça Roberlam, G., \& Luciano, M. N. (2015). Comparative performance analysis of a standard three-phase induction motor and an asymmetric three-phase induction motor fed from a single-phase network. Electric Power Systems Research, 125, 211-219. 
[5] Guedes, A. S., Silva, S. M., de Jesus Cardoso Filho, B., \& Conceição, C. A. (2016). Evaluation of electrical insulation in three-phase induction motors and classification of failures using neural networks. Electric Power Systems Research, 140, 263-273.

[6] El-Kharashi, E., El-Dessouki, M., Massoud, J. G., Farid, A. W., \& Al-Ahmar, M. A. (2018). The use of the current complex factor to determine the precise output energy of the induction motor. Electric Power Systems Research, 154, 23-36.

[7] Phipps, J. K., Nelson, J. P., \& Sen, P. K. (1994). Power quality and harmonic distortion on distribution systems. IEEE transactions on industry applications, 30(2), 476-484.

[8] Grajales, J., Ramírez, J., \& Cadavid, D. (2004). Efectos de los armónicos en los motores de inducción: una revisión. Revista Facultad de Ingeniería Universidad de Antioquia, (31).

[9] Valencia, D., Quispe, E., \& Sousa, V. (2014). Estudio del efecto de la distorsión armónica de tensión sobre la operación del motor trifásico de inducción en estado estacionario. Energética, (43), 67-77.

[10] Martínez-Pañeda, E. (2016). MATLAB: Una herramienta para la didáctica del Método de los Elementos Finitos. UNIÓN, Revista Iberoamericana de Educación Matemática, (45), 242-268.

[11] Fornieles, F. (2009). Armónicos: Efectos, diagnóstico y soluciones. XV Jornadas de Conferencias de Ingeniería Electrónica del Campus de Terrassa, Universitat Politècnica de Catalunya.

[12] García Santamaría, C. (2017). Análisis espectral de señales para la detección de fallos de motores de inducción.

[13] A Suarez, J. A., Di Mauro, G. F., Anaut, D., \& Agüero, C. (2005). Análisis de la distorsión armónica y los efectos de atenuación y diversidad en áreas residenciales. Revista del IEEE América Latina, 3(5), 71-77.

[14] Bollen, M. H., Ribeiro, P. F., Larsson, E. A., \& Lundmark, C. M. (2008). Limits for voltage distortion in the frequency range 2 to $9 \mathrm{kHz}$. IEEE Transactions on Power Delivery, 23(3), 1481-1487.

[15] Sapena-Bano, A., Martinez-Roman, J., Puche-Panadero, R., Pineda-Sanchez, M., Perez-Cruz, J., \& Riera-Guasp, M. (2018). Induction machine model with space harmonics for fault diagnosis based on the convolution theorem. International Journal of Electrical Power \& Energy Systems, 100, 463-481.

[16] Arahal, M. R., Barrero, F., Ortega, M. G., \& Martin, C. (2016). Harmonic analysis of direct digital control of voltage inverters. Mathematics and Computers in Simulation, 130, 155-166.

[17] Arcila, J. D. (2010). Armónicos en sistemas eléctricos. ieb sa, 1-26.

[18] salazar, 1. h. c. (2003, january). Programa en Matlab para analisis y simulacion de armonicos. In Simposio Internacional sobre la Calidad de la Energía EléctricaSICEL(Vol. 2).

[19] Bañó, A. S. (2014). Desarrollo de técnicas avanzadas de diagnóstico de máquinas eléctricas válidas para cualquier régimen de funcionamiento (Doctoral dissertation, Universitat Politècnica de València).

[20] Hernández, J. U. V., \& Yepez, E. C. (2017). Detección de barras rotas en motores de inducción utilizando la STFT. jóvenes en la ciencia, 3(2), 2383-2387. 
[21] García Santamaría, C. (2017). Análisis espectral de señales para la detección de fallos de motores de inducción.

[22] Oviedo, S. J., Quiroga, J. E., \& Ordóñez Plata, G. (2014). Validación Experimental de la Metodología Motor Current Signature Analysis para un Motor de Inducción de 2 HP. Revista Facultad de Ingeniería Universidad de Antioquia, (70). 


\section{PARA CITAR EL ARTÍCULO INDEXADO.}

Román Vargas, W., Barreno Layedra, N., Martínez Nogales, J., \& Cachuput Gusñay, J. (2019). Análisis de armónicos de un motor trifásico mediante transformadas integrales para analizar fallas de funcionamiento.

Ciencia

Digital,

3(3.4.),

$249-262$

https://doi.org/10.33262/cienciadigital.v3i3.4.869

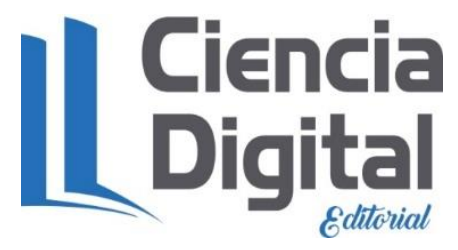

El artículo que se publica es de exclusiva responsabilidad de los autores y no necesariamente reflejan el pensamiento de la Revista Ciencia Digital.

El artículo queda en propiedad de la revista y, por tanto, su publicación parcial y/o total en otro medio tiene que ser autorizado por el director de la Revista Ciencia Digital.
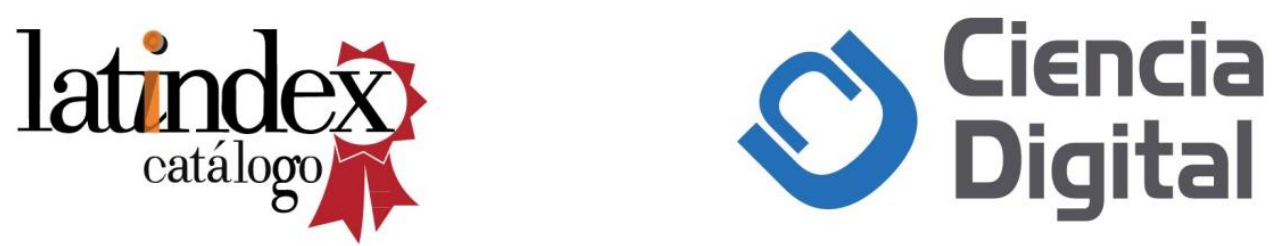